\title{
ASYMPTOTICS OF POSTERIORS FOR BINARY BRANCHING PROCESSES
}

\author{
DIDIER PIAU, ${ }^{*}$ Université Joseph Fourier
}

\begin{abstract}
We compute the posterior distributions of the initial population and parameter of binary branching processes in the limit of a large number of generations. We compare this Bayesian procedure with a more naïve one, based on hitting times of some random walks. In both cases, central limit theorems are available, with explicit variances.
\end{abstract}

Keywords: Branching process; Bayesian estimation; Polymerase chain reaction

2000 Mathematics Subject Classification: Primary 60J80

Secondary 92D25; 62F15; 60J85

\section{Introduction}

This paper is devoted to some estimation procedures of binary branching processes in a Bayesian setting. To be more specific, let $\left(X_{n}\right)_{n \geq 0}$ denote a Galton-Watson process which starts from the initial population $X_{0} \geq 1$ and whose offspring is ruled by the distribution

$$
(1-U) \delta_{1}+U \delta_{2} \quad \text { with } 0<U<1
$$

where $\delta_{x}$ denotes the Dirac mass at $x$. This means that, at every generation, each individual dies and is replaced by one or two individuals with probability $1-U$ and $U$, respectively, independently of the fate of the other individuals, and that $X_{n}$ counts generation $n$.

In a Bayesian framework, the initial population $X_{0}$ and the offspring parameter $U$ are both random and unknown. To keep things simple, we also assume that $X_{0}$ and $U$ are independent, and we wish to estimate them from the observation of a finite path $x_{1: n}=\left(x_{k}\right)_{1 \leq k \leq n}$ of the process $X_{1: n}=\left(X_{k}\right)_{1 \leq k \leq n}$ up to a given time $n \geq 1$.

Well-known motivations for such a study are various biological settings where one observes $X_{1: n}$, but $X_{0}$ and $U$ are unknown. One example is the modeling of polymerase chain reactions. Probabilistic models of polymerase chain reactions were proposed and studied in Sun (1995), Weiss and von Haeseler (1995), (1997), Peccoud and Jacob (1996), Piau (2002), (2004), (2005), and Jagers and Klebaner (2003). Recently, Lalam and Jacob (2007) introduced and studied the Bayesian setting above; see also Lalam (2007). For other Bayesian approaches of branching processes, see Scott (1987), Prakasa Rao (1992), and Mendoza and Gutiérrez-Peña (2000), and, for the interesting model of a bisexual branching process, see Molina et al. (1998) for example. Finally, the idea of studying a branching process backwards, but to estimate its age rather than its initial population, is examined in Klebaner and Sagitov (2002).

In models of polymerase chain reactions and in similar contexts, the initial population $X_{0}$ is the size of a small sample, extracted at random from a much larger population. This suggests that the initial population $X_{0}$ should be Poisson distributed, say with parameter $\Lambda$. We assume

Received 18 July 2007; revision received 11 March 2008.

* Postal address: Institut Fourier UMR 5582, Université Joseph Fourier Grenoble 1, 100 rue des Maths, BP 74, 38402 Saint Martin d'Hères, France. Email address: didier.piau@ujf-grenoble.fr 
that $\Lambda$ is random as well. Jeffreys' principle (see Kass and Wasserman (1996)) then indicates that the prior distributions of $\Lambda$ and $U$ should be proportional to measures which we compute below. To sum up the result of these computations, the prior of $\Lambda$ is easy to write down but improper and the prior of $U$ is awkward but proper. However, the posterior of $\left(X_{0}, U\right)$ conditionally on $X_{1: n}$ is a proper distribution, which can be computed explicitly. In particular, this posterior distribution depends only on $X_{1}, X_{n}$, and $S_{n}=X_{1}+\cdots+X_{n}$. Unfortunately, it is also rather unwieldy.

In such situations, one may rely on numerical algorithms, based on Markov chain Monte Carlo methods for example, to simulate the posterior distributions with any prescribed degree of accuracy. Instead, we look for simple asymptotics in realistic regimes. Namely, we assume that $n$ is large and we are interested in the asymptotic posterior distribution of $\left(X_{0}, U\right)$, assuming that $X_{n}$ is large and that the ratio $S_{n} / X_{n}$ converges to a finite limit. This assumption is almost surely fulfilled by the paths of binary branching processes, since these are supercritical. In this setting we show that the posterior distributions indeed converge and we compute their limit explicitly.

\section{Results}

To describe our results, we introduce some notation. Let $x_{0: \infty}=\left(x_{n}\right)_{n \geq 0}$ denote a sequence of positive integers. We say that such a sequence is admissible if, for every nonnegative $n$, $x_{n} \leq x_{n+1} \leq 2 x_{n}$. We say that an admissible sequence is regular if, furthermore, $x_{n} / s_{n}$ converges to a positive limit when $n$ goes to $\infty$, where $s_{n}=x_{1}+\cdots+x_{n}$. The binary index $B\left(x_{0: \infty}\right)$ of a regular admissible sequence $x_{0: \infty}$ is the real number in $(0,1]$ defined by

$$
B\left(x_{0: \infty}\right)=\lim _{n \rightarrow \infty} \frac{x_{n+1}}{s_{n}} .
$$

The renormalized index $R\left(x_{0: \infty}\right)$ of a regular admissible sequence $x_{0: \infty}$ is the real number in $[0,+\infty)$ defined by

$$
R\left(x_{0: \infty}\right)=\lim _{n \rightarrow \infty} \frac{\left(s_{n}-x_{n+1}\right)^{2}}{4 x_{n+1} s_{n}} .
$$

Almost every (sequence which can be realized as a) path of a binary branching process is admissible and regular. The renormalized index is a function of the binary index, namely $R\left(x_{0: \infty}\right)=\varrho\left(B\left(x_{0: \infty}\right)\right)$, where, for every $u$ in $(0,1]$,

$$
\varrho(u)=\frac{(1-u)^{2}}{4 u} .
$$

The binary index and the normalized index are asymptotic quantities, in the sense that, for every nonnegative integer $n$, the indexes of a regular admissible sequence $x_{0: \infty}$ do not depend on the first values, $x_{0: n}$.

From now on, letters $k$ and $n$ are used to enumerate generations of the process (that is, the time) and the symbols $x, x_{k}, x_{n}$, and $y$ are used to measure population sizes.

Definition 1. (Distributions.) For every positive real number $r$ and every positive integer $x$, the finite discrete measure $v(r, x)$ and the discrete probability measure $\mu(r, x)$, both on the positive integers, are defined by

$$
\nu(r, x)=\sum_{y=h(x)}^{x}\left(\begin{array}{c}
2 y \\
y
\end{array}\right)\left(\begin{array}{c}
y \\
x-y
\end{array}\right) r^{y} \delta_{y}, \quad \mu(r, x)=\frac{v(r, x)}{|v(r, x)|} .
$$


For every positive integer $x$, the integer $h(x)$ in (1) is the upper half of $x$, that is, the smallest integer such that $2 h(x) \geq x$. In other words, $h(2 x)=h(2 x-1)=x$ for every positive integer $x$.

Our main result is as follows.

Theorem 1. (Posterior distributions.) (i) The path $X_{0: \infty}$ of a binary branching process with parameter $U$ is almost surely regular admissible and its binary index is almost surely $B\left(X_{0: \infty}\right)=U$.

(ii) Assume that the prior distribution of $\left(X_{0}, U\right)$ satisfies Jeffreys' principle. Then, for every regular admissible sequence $x_{1: \infty}$ with binary index $u=B\left(x_{1: \infty}\right)$ in $(0,1)$, the posterior distribution of $\left(X_{0}, U\right)$, conditionally on $X_{1: n}=x_{1: n}$, converges when $n$ goes to $\infty$ to the distribution $\mu\left(\varrho(u), x_{1}\right) \otimes \delta_{u}$.

Theorem 1 shows that the limit posterior distribution of $X_{0}$ when $n$ goes to $\infty$ is almost surely $\mu(r, x)$ with $r=\varrho(U)$ and $x=X_{1}$. Unless $r=0, r=1$, or $x=1, \mu(r, x)$ is not degenerate; hence, the value of $X_{0}$ can be determined only with some uncertainty, even from an infinite trajectory $X_{1: \infty}$. On the contrary, $U$ is a function of the infinite trajectory $X_{1: \infty}$.

The limit distribution $\mu\left(\varrho(u), x_{1}\right) \otimes \delta_{u}$ in Theorem 1 converges to the Dirac distribution at $\left(x_{1}, 0\right)$ when $u$ converges to 0 and to the Dirac distribution at $\left(h\left(x_{1}\right), 1\right)$ when $u$ converges to 1 . Our next result describes the intuitively obvious variations of $\mu(r, x)$ with respect to $r$ and $x$. First, since $r=\varrho(u)$ is a decreasing function of $u$ and the offspring distribution of the branching process is stochastically increasing with $u$, we should expect $\mu(r, x)$ to increase stochastically when $r$ increases. Likewise, since $x$ represents the population at time 1, we should expect $\mu(r, x)$, which represents the population at time 0 , to increase stochastically when $x$ increases.

We recall that a measure $\mu_{1}$ is stochastically larger than a measure $\mu_{2}$ if and only if $\mu_{1}([z,+\infty)) \geq \mu_{2}([z,+\infty))$ for every real number $z$.

Proposition 1. (Ordering of limit posterior distributions.) For every positive integer $x$, the family $(\mu(r, x))_{r \geq 0}$ is stochastically increasing. For every positive real number $r$, the family $(\mu(r, x))_{x \geq 1}$ is stochastically increasing.

We now characterize the limit of $\mu(r, x)$ for every fixed value of $r$, when $x$ converges to $\infty$.

Theorem 2. (Limit posterior distributions of initial populations.) Fix $u$ in $(0,1)$. For every positive integer $x$, let $\xi_{x}$ denote a random variable with distribution $\mu(\varrho(u), x)$. When $x$ converges to $\infty$, the expectation and the mode of $\xi_{x} / x$ both converge to

$$
m_{u}=\frac{1}{1+u}
$$

and the random variables $\left(\xi_{x}-m_{u} x\right) / \sqrt{x}$ converge in distribution to a centered Gaussian distribution with variance

$$
\sigma_{u}^{2}=\frac{u(1-u)}{(1+u)^{3}}
$$

For the sake of comparison, we turn to another natural way to estimate initial populations of branching processes with known offspring distributions, based on hitting times. To describe this in the setting of binary branching processes, we first introduce some notation. 
Definition 2. (Hitting times.) Fix a real number $u$ in $(0,1)$, and let $\left(\varepsilon_{x}\right)_{x \geq 1}$ denote a sequence of independent Bernoulli random variables with distribution $(1-u) \delta_{1}+u \delta_{2}$. For every positive integer $x$, let $\sigma_{x}:=\varepsilon_{1}+\cdots+\varepsilon_{x}$. Define the distribution of the hitting time $\eta_{x}$ by the relation

$$
\mathrm{P}\left(\eta_{x}=y\right)=\mathrm{P}\left(\sigma_{y}=x \mid H_{x}\right), \quad \text { where } H_{x}=\left\{\text { there exists } z \geq 1 ; \sigma_{z}=x\right\} .
$$

When the value of $u$ is known, an estimation procedure of $X_{0}$ based on $X_{1}=x$ is to propose the value $y$ for $X_{0}$ with probability $\mathrm{P}\left(\eta_{x}=y\right)$; thus, an estimator of $X_{0}$, when $X_{1}=x$, is the distribution of $\eta_{x}$.

Recall that $m_{u}=1 /(1+u)$ and $\sigma_{u}^{2}=u(1-u) /(1+u)^{3}$.

Theorem 3. (Initial populations through hitting times.) Fix a real number $u$ in $(0,1)$. For every positive integer $x$,

$$
\left|\mathrm{E}\left(\eta_{x}\right)-m_{u} x\right| \leq \frac{2 u}{(1+u)^{2}} \leq \frac{1}{2} .
$$

Furthermore, when $x$ converges to $\infty,\left(\eta_{x}-m_{u} x\right) / \sqrt{x}$ converges in distribution to a centered Gaussian variable with variance $\sigma_{u}^{2}$.

The rest of the paper is organized as follows. We prove Theorem 1 and Proposition 1 in Section 3, and Theorem 2 in Section 4. Finally, the proof of Theorem 3, sharper bounds on $\mathrm{E}\left(\eta_{x}\right)$, and a brief comparison with another, non-Bayesian, estimation procedure are given in Section 5 .

\section{Posterior distributions}

\subsection{Preliminaries}

Jeffreys' principle (see Kass and Wasserman (1996)) indicates that the prior measure for a parameter $\theta$ governing the distribution $v_{\theta}$ of a random variable $Z$ should have a density proportional to $J(\theta)^{1 / 2}$, where

$$
J(\theta)=-\mathrm{E}_{\theta}\left(\frac{\partial^{2}}{\partial \theta^{2}} \log v_{\theta}(Z)\right)
$$

We apply this to the parameter $(\Lambda, U)$. Parts of Lemma 1, below, are given in Lalam and Jacob (2007).

Lemma 1. For every positive integer $n$, the prior measure for $(\Lambda, U)$ according to Jeffreys' principle and based on $X_{0: n}$ is the product of the prior measures for $\Lambda$ and $U$. The prior measures for $\Lambda$ and $U$ are respectively proportional to the measures $\mathrm{d} \lambda / \sqrt{\lambda}$ on $\lambda>0$ and $\pi_{n}(u) \mathrm{d} u$ on $0<u<1$, where

$$
\pi_{n}(u)=\sqrt{\frac{(1+u)^{n}-1}{u^{2}(1-u)}} .
$$

In particular, the prior of $U$ is proper.

Proof. Assume that $X_{0}$ is Poisson distributed with parameter $\Lambda$ and that $X_{0: n}$ is a binary branching process with parameter $U$. Then the distribution $v_{\Lambda, U}$ of $X_{0: n}$ is such that

$$
v_{\Lambda, U}\left(x_{0: n}\right)=\mathrm{e}^{-\Lambda} \frac{\Lambda^{x_{0}}}{x_{0} !} \prod_{k=1}^{n}\left(\begin{array}{c}
x_{k-1} \\
x_{k}-x_{k-1}
\end{array}\right) U^{x_{k}-x_{k-1}}(1-U)^{2 x_{k-1}-x_{k}} .
$$


Up to a factor $C\left(x_{0: n}\right)$, which does not depend on $(\Lambda, U), \log v_{\Lambda, U}\left(x_{0: n}\right)$ is

$$
-\Lambda+x_{0} \log \Lambda+\left(x_{n}-x_{0}\right) \log U+\left(s_{n}-2 x_{n}+2 x_{0}\right) \log (1-U)+C\left(x_{0: n}\right) .
$$

This is the sum of a function of $\Lambda$ and a function of $U$; hence, the prior measures are product measures. As regards the prior for $\Lambda$,

$$
\frac{\partial^{2}}{\partial \Lambda^{2}} \log v_{\Lambda}\left(x_{0: n}\right)=-\frac{x_{0}}{\Lambda^{2}} ; \quad \text { hence, } \quad J(\Lambda)=\frac{\mathrm{E}_{\Lambda}\left(X_{0}\right)}{\Lambda^{2}}=\frac{1}{\Lambda} .
$$

As regards the prior for $U$,

$$
\frac{\partial^{2}}{\partial U^{2}} \log v_{U}\left(x_{0: n}\right)=-\frac{x_{n}-x_{0}}{U^{2}}-\frac{s_{n}-2 x_{n}+2 x_{0}}{(1-U)^{2}} ;
$$

hence,

$$
J_{n}(U)=\frac{\mathrm{E}_{U}\left(X_{n}-X_{0}\right)}{U^{2}}+\frac{\mathrm{E}_{U}\left(S_{n}-2 X_{n}+2 X_{0}\right)}{(1-U)^{2}},
$$

where $S_{n}=X_{1}+\cdots+X_{n}$. Since $\mathrm{E}_{U}\left(X_{k}\right)=(1+U)^{k} \mathrm{E}\left(X_{0}\right)$ for every nonnegative integer $k$, we find that $J_{n}(U)=\mathrm{E}\left(X_{0}\right) \pi_{n}(U)^{2}$, using the notation of the lemma.

Finally, up to multiplicative constants, $\pi_{n}(u)$ behaves like $1 / \sqrt{u}$ when $u$ converges to 0 and like $1 / \sqrt{1-u}$ when $u$ converges to 1 . Hence, $\pi_{n}$ is integrable and there exists a (proper) prior distribution for $U$. This completes the proof.

From now on, we fix a positive integer $n$, we assume that the observations are $X_{1: n}=x_{1: n}$ with $x_{1: n}=\left(x_{k}\right)_{1 \leq k \leq n}$, and we recall that $s_{n}=x_{1}+\cdots+x_{n}$. The posterior distribution in Lemma 2, below, is similar, but not equal, to a posterior distribution computed in Lalam and Jacob (2007).

Lemma 2. The posterior distribution of $\left(X_{0}, U\right)$, conditionally on $X_{1: n}=x_{1: n}$, depends only on $x_{1}, x_{n}$, and $s_{n}$, and is proportional to the measure

$$
\sum_{x=h\left(x_{1}\right)}^{x_{1}} 2^{-2 x}\left(\begin{array}{c}
2 x \\
x
\end{array}\right)\left(\begin{array}{c}
x \\
x_{1}-x
\end{array}\right) u^{x_{n}-x}(1-u)^{s_{n}-2 x_{n}+2 x} \pi_{n}(u) \delta_{x} \otimes \mathrm{d} u .
$$

Proof. Fix $u, x_{1: n}$, and $x$ such that $h\left(x_{1}\right) \leq x \leq x_{1}$. Then, the conditional probability $\mathrm{P}\left(U \in \mathrm{d} u, X_{0}=x \mid X_{1: n}=x_{1: n}\right)$ is proportional to

$$
v_{U}(\mathrm{~d} u) \int v_{\Lambda}(\mathrm{d} \lambda) \mathrm{P}_{\lambda}\left(X_{0}=x\right) \mathrm{P}_{u}\left(X_{1: n}=x_{1: n} \mid X_{0}=x\right),
$$

where $v_{U}(\mathrm{~d} u)=\pi_{n}(u) \mathrm{d} u$ and $v_{\Lambda}(\mathrm{d} \lambda)=\mathrm{d} \lambda / \sqrt{\lambda}$. Hence,

$$
\int v_{\Lambda}(\mathrm{d} \lambda) \mathrm{P}_{\lambda}\left(X_{0}=x\right)=\frac{\Gamma(x+1 / 2)}{\Gamma(x+1)}=\sqrt{\pi} 2^{-2 x}\left(\begin{array}{c}
2 x \\
x
\end{array}\right) .
$$

Likewise, using the computations in the proof of Lemma 1, we obtain

$$
\mathrm{P}_{u}\left(X_{1: n}=x_{1: n} \mid X_{0}=x\right)=C\left(x_{1: n}\right)\left(\begin{array}{c}
x \\
x_{1}-x
\end{array}\right) u^{x_{n}-x}(1-u)^{s_{n}-2 x_{n}+2 x},
$$

where $C\left(x_{1: n}\right)$ does not depend on $(x, u)$. This completes the proof. 


\subsection{Proof of Theorem 1}

Part (i) follows from the fact that, when $n$ converges to $\infty, X_{n} /(1+U)^{n}$ converges almost surely to a random positive and finite limit.

A sketch of the proof of part (ii) is as follows. Consider the distribution in Lemma 2, and assume that $x_{n}$ converges to $\infty$ and that $x_{n} /\left(s_{n}-x_{n}\right)$ converges to $v$. Then $s_{n}-x_{n}$ is equivalent to $x_{n} / v$; hence,

$$
u^{x_{n}}(1-u)^{s_{n}-2 x_{n}}=\left(u^{v}(1-u)^{1-v}\right)^{S_{n}-x_{n}+o\left(x_{n}\right)} .
$$

The inner parenthesis is maximal when $u=v$, and the exponent converges to $\infty$; hence, this contribution becomes concentrated around the value $u=v$. The remaining factor involving $u$ in the distribution described in Lemma 2 is $\varrho(u)^{x}$, and the convergence to $\mu\left(\varrho(v), x_{1}\right)$ follows.

For a detailed proof of part (ii), we consider a sequence $x_{1: \infty}$ such that $x_{n}$ converges to $\infty$ and $x_{n} /\left(s_{n}-x_{n}\right)$ converges to $v$. For every positive integer $n$, we introduce random variables $\left(T_{n}, U_{n}\right)$ distributed as $\left(X_{0}, U\right)$ conditionally on $X_{1: n}=x_{1: n}$. We first show the convergence in probability of $U_{n}$, then the convergence in distribution of $\left(T_{n}, U_{n}\right)$.

Lemma 3. With the notation above, $U_{n}$ converges to $v$ in probability.

Proof. Lemma 2 yields

$$
\mathrm{P}\left(T_{n}=x, U_{n} \in \mathrm{d} u\right)=c_{n} p_{x} \varrho(u)^{x} b_{n}(u) q_{n}(u) \mathrm{d} u,
$$

where $c_{n}$ denotes a normalizing constant which is independent of $x$ and $u, p_{x}$ depends only on $x$ and $x_{1}, b_{n}(u)$ depends only on $u, x_{n}$, and $s_{n}$, and $q_{n}(u)$ depends only on $u$ and $n$. More precisely, for every integer $x$ such that $x_{1} \leq 2 x \leq 2 x_{1}$ and every real number $u$ in $(0,1)$,

$$
\begin{aligned}
p_{x} & =\left(\begin{array}{c}
2 x \\
x
\end{array}\right)\left(\begin{array}{c}
x \\
x_{1}-x
\end{array}\right), \\
b_{n}(u) & =u^{x_{n}-1 / 2(1-u)^{S_{n}-2 x_{n}-1 / 2},} \\
q_{n}(u) & =\sqrt{\frac{(1+u)^{n}-1}{u}} .
\end{aligned}
$$

We aim to show that, for every integer $x$ such that $p_{x}$ is positive and every positive real number $z$, when $n$ converges to $\infty$,

$$
\mathrm{P}\left(T_{n}=x,\left|U_{n}-v\right| \geq z\right) \ll \mathrm{P}\left(T_{n}=x\right) .
$$

Since the function $q_{n}$ is nondecreasing,

$$
\mathrm{P}\left(T_{n}=x,\left|U_{n}-v\right| \geq z\right) \leq c_{n} p_{x} q_{n}(1) \int_{|u-v| \geq z} \varrho(u)^{x} b_{n}(u) \mathbf{1}_{[0,1]}(u) \mathrm{d} u
$$

and

$$
\mathrm{P}\left(T_{n}=x\right) \geq c_{n} p_{x} q_{n}(0) \int_{0}^{1} \varrho(u)^{x} b_{n}(u) \mathrm{d} u .
$$

The ratio of the two integrals written above is $\mathrm{P}\left(\left|B_{n}-v\right| \geq z\right)$, where $B_{n}$ is a beta random variable of parameters $\left(\alpha_{n}, \beta_{n}\right)$, with

$$
\alpha_{n}=x_{n}-x+\frac{1}{2}, \quad \beta_{n}=s_{n}-2 x_{n}+2 x+\frac{1}{2} .
$$


Since $\alpha_{n}$ and $\beta_{n}$ both converge to $\infty$ and $\alpha_{n} /\left(\alpha_{n}+\beta_{n}\right)$ converges to $v$, it is an easy matter to show that $B_{n}$ converges in probability to $v$. However, we need a stronger statement, namely, the fact that $\mathrm{P}\left(\left|B_{n}-v\right| \geq z\right) \ll q_{n}(0) / q_{n}(1)$. Note that $q_{n}(0)=\sqrt{n}$ and $q_{n}(1) \sim 2^{n / 2}$; hence, $q_{n}(0) / q_{n}(1) \ll 1$.

We can write an elementary proof of this, based on the representation of beta random variables with integer parameters as ratios of sums of independent and identically distributed exponential random variables and on large deviations properties of these sums. Instead, however, we rely on the approximations of beta distributions by normal distributions provided in Alfers and Dinges (1984). A rephrasing of Corollary 1 on page 405 of that paper is as follows. Let $\left(Y_{k}\right)_{k}$ denote a sequence of beta random variables of parameters $\left(k a_{k}, k\left(1-a_{k}\right)\right)$. Assume that $k$ converges to $\infty$ and that $a_{k}$ converges to a limit $0<a<1$. Then, for every fixed $y$ such that $a<y<1$, the ratio

$$
\frac{\mathrm{P}\left(Y_{k} \geq y\right)}{\mathrm{P}\left(Z \geq \sqrt{2 k \ell\left(a_{k}, y\right)}\right)}
$$

converges to a finite and positive limit, which depends on $a$ and $y$ only, where $Z$ denotes a standard Gaussian random variable and $\ell$ denotes the function defined by

$$
\ell(\alpha, y)=\alpha \log \left(\frac{\alpha}{y}\right)+(1-\alpha) \log \left(\frac{1-\alpha}{1-y}\right) .
$$

Since $a_{k}$ converges to $a$ and $\ell(\alpha, y)$ is a continuous function of $\alpha$, standard estimates of Gaussian tails and the result of Alfers and Dinges show that there exists a positive constant $C<1$, independent of $k$, such that, for every large enough $k$,

$$
\mathrm{P}\left(Y_{k} \geq y\right) \leq C^{k} .
$$

Applying this to our setting, first to the random variables $B_{n}$ and to $y=v+z$, then to the random variables $1-B_{n}$ and to $y=1-v+z$, we obtain the existence of a constant $C<1$ such that, for every large enough $n$,

$$
\mathrm{P}\left(\left|B_{n}-v\right| \geq z\right) \leq 2 C^{\alpha_{n}+\beta_{n}} .
$$

Since $\alpha_{n}+\beta_{n}=s_{n-1}+x+1 \geq s_{n-1} \gg n, 2 C^{\alpha_{n}+\beta_{n}} \ll q_{n}(0) / q_{n}(1)$. This completes the proof.

We now apply Lemma 3 to the proof of part (ii). Introduce the finite sums

$$
p(u)=\sum_{x} p_{x} \varrho(u)^{x} .
$$

For every $u$ in $(0,1)$, the distribution of $T_{n}$, conditionally on $U_{n}=u$, is independent of $n$ and such that

$$
\mathrm{P}\left(T_{n}=x \mid U_{n}=u\right)=p(u)^{-1} p_{x} \varrho(u)^{x} .
$$

Hence, for every measurable subset $B$ of $(0,1)$,

$$
\mathrm{P}\left(T_{n}=x, U_{n} \in B\right)=\mathrm{E}\left(p\left(U_{n}\right)^{-1} p_{x} \mathbf{1}_{B}\left(U_{n}\right) \varrho\left(U_{n}\right)^{x}\right) .
$$

The function $u \mapsto p(u)^{-1} p_{x} \mathbf{1}_{B}(u) \varrho(u)^{x}$ is bounded by 1 on $(0,1)$ and, as soon as $v$ is not in the boundary of $B$, continuous at $u=v$. Since $U_{n}$ converges in distribution to $v$, this implies that $\mathrm{P}\left(T_{n}=x, U_{n} \in B\right)$ converges to $p(v)^{-1} p_{x} \mathbf{1}_{B}(v) \varrho(v)^{x}$, for instance, for every interval $B=[0, u]$ with $u \neq v$. This is equivalent to the desired convergence in distribution. 


\subsection{Remarks}

For every positive integer $n$ and every admissible sample, $s_{n} \geq 2 x_{n}\left(1-1 / 2^{n}\right)$, since $x_{k} \geq$ $x_{k+1} / 2$ for every nonnegative integer $k$; hence, $s_{n}-x_{n} \geq x_{n}+o\left(x_{n}\right)$ and $u \leq 1$ in the asymptotics that we consider. Furthermore, the function $\varrho$ decreases from $\varrho\left(0^{+}\right)=+\infty$ to $\varrho\left(1^{-}\right)=0$.

The measures $\mu(r, x)$ for the first values of $x$ are as follows:

$$
\begin{gathered}
\mu(r, 1)=\delta_{1}, \quad \mu(r, 2)=\frac{\delta_{1}+3 r \delta_{2}}{1+3 r}, \quad \mu(r, 3)=\frac{3 \delta_{2}+5 r \delta_{3}}{3+5 r}, \\
\mu(r, 4)=\frac{3 \delta_{2}+30 r \delta_{3}+35 r^{2} \delta_{4}}{3+30 r+35 r^{2}}, \quad \text { and } \quad \mu(r, 5)=\frac{15 \delta_{3}+70 r \delta_{4}+63 r^{2} \delta_{5}}{15+70 r+63 r^{2}} .
\end{gathered}
$$

\subsection{Proof of Proposition 1}

The monotonicity with respect to $r$ is valid in a wider setting, described in Proposition 2, below, but the monotonicity with respect to $x$ is more specific.

Proposition 2. Let $\mu$ denote a nonzero bounded measure on the real line with exponential moments. For every real number $a$, introduce the measures $v_{a}$ and $\mu_{a}$ defined by the relations $v_{a}(\mathrm{~d} x)=\mathrm{e}^{a x} \mu(\mathrm{d} x)$ and $\mu_{a}=v_{a} /\left|v_{a}\right|$. Then the family $\left(\mu_{a}\right)_{a}$ is stochastically nondecreasing.

Proof. Fix $x$ in the real line. The derivative of $\mu_{a}([x,+\infty))$ with respect to $a$ has the sign of $D(x)$, where

$$
D(x)=\int_{y \geq x} y \mathrm{e}^{a y} \mu(\mathrm{d} y) \int \mathrm{e}^{a z} \mu(\mathrm{d} z)-\int_{y \geq x} \mathrm{e}^{a y} \mu(\mathrm{d} y) \int z \mathrm{e}^{a z} \mu(\mathrm{d} z) .
$$

The variations of $D(x)$ with respect to $x$ are given by

$$
\mathrm{d} D(x)=\mathrm{e}^{a x} \mu(\mathrm{d} x) \int(y-x) \mathrm{e}^{a y} \mu(\mathrm{d} y) .
$$

The integral on the right-hand side is a nonincreasing function of $x$. Since $D(-\infty)=$ $D(+\infty)=0$, the function $x \mapsto D(x)$ is nondecreasing for $x \leq x_{a}$ and nonincreasing for $x \geq x_{a}$, where $x_{a}$ solves the equation

$$
\int y \mathrm{e}^{a y} \mu(\mathrm{d} y)=x_{a} \int \mathrm{e}^{a y} \mu(\mathrm{d} y) .
$$

This proves that $D(x) \geq 0$ for every $x$; hence, $\mu_{a}([x,+\infty)) \leq \mu_{b}([x,+\infty))$ for every $a \leq b$. This completes the proof.

We turn to the monotonicity of $\mu(r, x)$ with respect to $x$. We fix a value of $r$ and write every $v(r, x)$ as

$$
v(r, x)=\sum_{y} a_{y}^{x} \delta_{y} .
$$

We want to prove that, for every fixed $x, G(z) \geq 0$ for every $z$, where

$$
G(z)=\sum_{y} a_{y}^{x} \sum_{y \geq z} a_{y}^{x+1}-\sum_{y \geq z} a_{y}^{x} \sum_{y} a_{y}^{x+1} .
$$


We see that $G(0)=G(\infty)=0$, and simple computations show that

$$
F(z)=\frac{1}{a_{z}^{x}}(G(z+1)-G(z))=\sum_{y} a_{y}^{x+1}-\frac{a_{z}^{x+1}}{a_{z}^{x}} \sum_{y} a_{y}^{x} .
$$

At this point, we use the specific form of the coefficients $a_{z}^{x}$, which yields

$$
\frac{a_{z}^{x+1}}{a_{z}^{x}}=2 \frac{(2 x+1)(2 z-x)}{(x+1)(x+1-z)} .
$$

This shows that $(F(z))_{z}$ is a nonincreasing sequence; hence, $G(z+1)-G(z) \geq 0$ if $z<z_{*}$ and $G(z+1)-G(z) \leq 0$ if $z \geq z_{*}$ for a given $z_{*}$. Hence, the sequence $(G(z))_{z}$ is nondecreasing on $z \leq z_{*}$ and nonincreasing on $z \geq z_{*}$. Since $G(0)=G(\infty)=0$, this implies that $G(z) \geq 0$ for every positive $z$. This completes the proof.

\section{Limit posterior distributions of initial populations}

\subsection{Expectations}

Fix $u$ in $(0,1)$, and let $r=\varrho(u)$. We are interested in the limit as $x \rightarrow \infty$ of the sequence

$$
\frac{1}{x} \mathrm{E}\left(\xi_{x}\right)=\frac{A(r, x)}{x B(r, x)},
$$

where

$$
A(r, x)=\sum_{y} y v(r, x)(y)=\sum_{y} y\left(\begin{array}{c}
2 y \\
y
\end{array}\right)\left(\begin{array}{c}
y \\
x-y
\end{array}\right) r^{y}
$$

and

$$
B(r, x)=|v(r, x)|=\sum_{y}\left(\begin{array}{c}
2 y \\
y
\end{array}\right)\left(\begin{array}{c}
y \\
x-y
\end{array}\right) r^{y} .
$$

Definition 3. For every positive $\lambda$ and $r$, introduce

$$
C_{\lambda}(r, z)=(1-4 r z(1+z))^{-\lambda}=\sum_{x \geq 0} c_{\lambda}(r, x) z^{x} .
$$

Starting from the expansion

$$
(1-4 z)^{-1 / 2}=\sum_{x \geq 0}\left(\begin{array}{c}
2 x \\
x
\end{array}\right) z^{x}
$$

we can write $B(r, x)$ as the coefficient of $z^{x}$ in the expansion of $C_{1 / 2}(r, x)$ along the powers of $z$, namely, $B(r, x)=c_{1 / 2}(r, x)$. Likewise, $A(r, x)$ is $r$ times the derivative of $B(r, x)$ with respect to $r$; hence, $A(r, x)$ is the coefficient of $z^{x}$ in the expansion of $2 r z(1+z) C_{3 / 2}(r, x)$ along the powers of $z$. This yields

$$
A(r, x)=2 r\left(c_{3 / 2}(r, x-1)+c_{3 / 2}(r, x-2)\right)
$$

and

$$
x B(r, x)=2 r\left(c_{3 / 2}(r, x-1)+2 c_{3 / 2}(r, x-2)\right) .
$$


Definition 4. For every positive $r$, introduce

$$
\gamma(r)=\frac{1}{2}\left(\sqrt{\frac{1+r}{r}}-1\right), \quad m(r)=\frac{1+\gamma(r)}{1+2 \gamma(r)}=\frac{1}{2}\left(1+\sqrt{\frac{r}{1+r}}\right) .
$$

Note that, for every $u$ in $(0,1)$,

$$
\gamma(\varrho(u))=\frac{u}{1-u}, \quad m(\varrho(u))=\frac{1}{1+u}=m_{u} .
$$

Lemma 4. For every positive $\lambda$ and $r$, when $x$ converges to $\infty$,

$$
c_{\lambda}(r, x) \sim c_{\lambda}(r) x^{\lambda-1} \gamma(r)^{-x}, \quad c_{\lambda}(r)=\frac{m(r)^{\lambda}}{\Gamma(\lambda)} .
$$

Proof. This is a consequence of known expansions of powers of $1 /(1-z)$. First, recall that

$$
(1-z)^{-\lambda}=\sum_{x \geq 0} d_{\lambda}(x) z^{x}, \quad d_{\lambda}(x)=\frac{\Gamma(x+\lambda)}{\Gamma(x+1) \Gamma(\lambda)} \sim \frac{x^{\lambda-1}}{\Gamma(\lambda)} .
$$

We use this and the decomposition

$$
1-4 r z(1+z)=\left(1-\frac{z}{\gamma(r)}\right)\left(1+\frac{z}{\gamma(r)+1}\right)
$$

to obtain the expansion

$$
C_{\lambda}(r, z)=\sum_{x} d_{\lambda}(x)\left(\frac{z}{\gamma(r)}\right)^{x} \sum_{x} d_{\lambda}(x)\left(\frac{-z}{1+\gamma(r)}\right)^{x},
$$

which implies that

$$
c_{\lambda}(r, x)=d_{\lambda}(x) \gamma(r)^{-x} \sum_{y=0}^{x}\left(\frac{-\gamma(r)}{\gamma(r)+1}\right)^{y} d_{\lambda}(y) \frac{d_{\lambda}(x-y)}{d_{\lambda}(x)} .
$$

When $x$ converges to $\infty$, the ratios $d_{\lambda}(x-y) / d_{\lambda}(x)$ converge to 1 ; hence, by dominated convergence,

$$
c_{\lambda}(r, x) \sim d_{\lambda}(x) \gamma(r)^{-x} \sum_{y \geq 0}\left(\frac{-\gamma(r)}{\gamma(r)+1}\right)^{y} d_{\lambda}(y)=d_{\lambda}(x) \gamma(r)^{-x}\left(1+\frac{\gamma(r)}{1+\gamma(r)}\right)^{-\lambda},
$$

where the equality stems from the definition of the coefficients $d_{\lambda}(\cdot)$. Substituting the equivalent of $d_{\lambda}(x)$ into this and using the fact that $1+\gamma(r) /(1+\gamma(r))=1 / m(r)$, we deduce Lemma 4 .

Lemma 4 for $\lambda=\frac{3}{2}$ yields, when $x$ converges to $\infty$, a constant $\alpha$, whose value is irrelevant, such that

$$
A(r, x) \sim 2 r \alpha x^{1 / 2} \gamma(r)^{-x} \gamma(r)(1+\gamma(r))
$$

and

$$
x B(r, x)-A(r, x) \sim 2 r \alpha x^{1 / 2} \gamma(r)^{-x} \gamma(r)^{2} .
$$


Hence, $(x B(r, x)-A(r, x)) / A(r, x)$ converges to $\gamma(r) /(1+\gamma(r))$ and

$$
\frac{A(r, x)}{x B(r, x)} \text { converges to } \frac{1+\gamma(r)}{1+2 \gamma(r)}=m(r) \text {. }
$$

This is the desired convergence of the expectations because, as mentioned above, the relation $r=\varrho(u)$ means that $m(r)=m_{u}$.

\subsection{Modes}

To study the mode of $\xi_{x}$, we compare $v(r, x)(y+1)$ to $v(r, x)(y)$. The ratios

$$
\frac{v(r, x)(y+1)}{v(r, x)(y)}=\frac{(y+1 / 2)(x-y) r}{(y+1-x / 2)(y+1 / 2-x / 2)}
$$

are the terms of a nonincreasing sequence indexed by $y$. Writing $y$ as $y=x(1+s) / 2 s$ with $s \geq 1$, when $x$ is large, we obtain

$$
\frac{v(r, x)(y+1)}{v(r, x)(y)} \sim r\left(s^{2}-1\right) .
$$

This implies that the sequence $(v(r, x)(y))_{y}$ is increasing on $y \leq y_{*}$ and decreasing on $y \geq y_{*}$ for a value of $y_{*}$ such that $y_{*}=x\left(1+s_{*}\right) / 2 s_{*}+o(x)$ with $s_{*}^{2}=1+1 / r$. Finally, this shows that, when $r=\varrho(u)$, the mode of $\mu(r, x)$ is at $x /(1+u)+o(x)$.

\subsection{Distributions}

Our next computation is based on characteristic functions. Fix $u$ in $(0,1)$, and let $r=\varrho(u)$. For every positive integer $x$, introduce

$$
F_{x}(t)=\mathrm{E}\left(\exp \left(t \frac{\xi_{x}-x m}{\sqrt{x}}\right)\right)
$$

Recall that

$$
m(r)=\frac{1+\gamma(r)}{1+2 \gamma(r)}, \quad \frac{1}{1+2 \gamma(r)}=\sqrt{\frac{r}{1+r}}, \quad r=\frac{(1-u)^{2}}{4 u} .
$$

Since $\mathrm{E}\left(\exp \left(t \xi_{x}\right)\right)=B\left(r \mathrm{e}^{t}, x\right) / B(r, x)$,

$$
F_{x}(t)=\frac{\mathrm{e}^{-t \sqrt{x} m} B\left(r \mathrm{e}^{t / \sqrt{x}}, x\right)}{B(r, x)} .
$$

We turn to the study of the sequence of functions $(B(\cdot, x))_{x \geq 1}$.

Since $B(r, x)=c_{1 / 2}(r, x)$, a consequence of Lemma 4 is that, when $x$ converges to $\infty$,

$$
\frac{B\left(r \mathrm{e}^{t / \sqrt{x}}, x\right)}{B(r, x)} \sim\left(\frac{\gamma(r)}{\gamma\left(r \mathrm{e}^{t / \sqrt{x}}\right)}\right)^{x} \frac{S(x, t / \sqrt{x})}{m(r)^{1 / 2}},
$$

where, for every $s$,

$$
S(x, s)=\sum_{y=0}^{x}\left(\frac{-\gamma\left(r \mathrm{e}^{s}\right)}{\gamma\left(r \mathrm{e}^{s}\right)+1}\right)^{y} d_{1 / 2}(y) \frac{d_{1 / 2}(x-y)}{d_{1 / 2}(x)} .
$$

We get rid of the fraction involving $S(x, t / \sqrt{x})$ through Lemmas 5 and 6 , below. 
Lemma 5. For every nonnegative $x$ and $y, d_{1 / 2}(y) d_{1 / 2}(x) \leq d_{1 / 2}(x+y)$.

Proof. A probabilistic proof is as follows. For every nonnegative $x, d_{1 / 2}(x)=2^{-2 x}\left(\begin{array}{c}2 x \\ x\end{array}\right)$ is the probability that a simple symmetric random walk on the integer line is at its starting point after $2 x$ steps. Hence, $d_{1 / 2}(x+y)$ is the probability that the random walk is at its starting point after $2 x+2 y$ points and $d_{1 / 2}(y) d_{1 / 2}(x)$ is the probability that the random walk is at its starting point after $2 x$ steps and also after $2 x+2 y$ points. The latter event being included in the former, showing the desired inequality.

Lemma 6. When $x$ converges to $\infty, S(x, t / \sqrt{x})$ converges to $m(r)^{1 / 2}$.

Proof. Since $S(x, 0)$ converges to $S(\infty, 0)=m(r)^{1 / 2}$ when $x$ converges to $\infty$, we show that $S(x, t / \sqrt{x})-S(x, 0)$ converges to 0 . By Lemma 5 , the ratios of coefficients $d_{1 / 2}$ involved in $S(x, t / \sqrt{x})$ and $S(x, 0)$ are bounded by 1 . Adding terms such that $y \geq x+1$, we obtain $|S(x, t / \sqrt{x})-S(x, 0)| \leq T(t / \sqrt{x})$, where

$$
T\left(\frac{t}{\sqrt{x}}\right)=\sum_{y=0}^{+\infty}\left|\left(\frac{\gamma\left(r \mathrm{e}^{t / \sqrt{x}}\right)}{\gamma\left(r \mathrm{e}^{t / \sqrt{x}}\right)+1}\right)^{y}-\left(\frac{\gamma(r)}{\gamma(r)+1}\right)^{y}\right| .
$$

All the terms in the sum have the same sign; hence,

$$
T\left(\frac{t}{\sqrt{x}}\right)=\left|\sum_{y=0}^{+\infty}\left(\frac{\gamma\left(r \mathrm{e}^{t / \sqrt{x}}\right)}{\gamma\left(r \mathrm{e}^{t / \sqrt{x}}\right)+1}\right)^{y}-\left(\frac{\gamma(r)}{\gamma(r)+1}\right)^{y}\right| .
$$

We can compute the sum of each geometric series. This yields

$$
\left|S\left(x, \frac{t}{\sqrt{x}}\right)-S(x, 0)\right| \leq T\left(\frac{t}{\sqrt{x}}\right)=\left|\gamma\left(r \mathrm{e}^{t / \sqrt{x}}\right)-\gamma(r)\right|,
$$

which proves the lemma since $\gamma(\cdot)$ is a continuous function.

Lemma 6 above shows that

$$
\frac{B\left(r \mathrm{e}^{t / \sqrt{x}}, x\right)}{B(r, x)} \sim\left(\frac{\gamma\left(r \mathrm{e}^{t / \sqrt{x}}\right)}{\gamma(r)}\right)^{-x} .
$$

The rest of the proof is standard. A Taylor expansion of $\gamma(\cdot)$ around $r$ yields

$$
\gamma\left(r \mathrm{e}^{t / \sqrt{x}}\right)=\gamma(r)+\left(\mathrm{e}^{t / \sqrt{x}}-1\right) \gamma^{\prime}(r)+\frac{\left(\mathrm{e}^{t / \sqrt{x}}-1\right)^{2} \gamma^{\prime \prime}(r)}{2}+o\left(\left(\mathrm{e}^{t / \sqrt{x}}-1\right)^{2}\right) .
$$

Using the expansion of $\mathrm{e}^{t / \sqrt{x}}$ along powers of $1 / \sqrt{x}$ and dividing everything by $\gamma(r)$, we obtain

$$
\frac{\gamma\left(r \mathrm{e}^{t / \sqrt{x}}\right)}{\gamma(r)}=1+\left(r \frac{\gamma^{\prime}(r)}{\gamma(r)}\right) \frac{t}{\sqrt{x}}+\left(r \frac{\gamma^{\prime}(r)}{\gamma(r)}+r^{2} \frac{\gamma^{\prime \prime}(r)}{\gamma(r)}\right) \frac{t^{2}}{2 x}+o\left(\frac{1}{x}\right) \text {. }
$$

Note that

$$
r \frac{\gamma^{\prime}(r)}{\gamma(r)}=-m(r)
$$

Taking logarithms, writing the ratio of functions $\gamma$ as

$$
\left(\frac{\gamma\left(r \mathrm{e}^{t / \sqrt{x}}\right)}{\gamma(r)}\right)^{-x}=\exp \left(-x \log \left(\frac{\gamma\left(r \mathrm{e}^{t / \sqrt{x}}\right)}{\gamma(r)}\right)\right)
$$


and using the expansion $\log (1+z)=z-z^{2} / 2+o\left(z^{2}\right)$ when $z=o(1)$, we find that $F_{x}(t)$ is equivalent to the exponential of

$$
-t m(r) \sqrt{x}-x\left(-\frac{m(r) t}{\sqrt{x}}+\frac{\left(m_{2}(r)-m(r)\right) t^{2}}{2 x}-\frac{m(r)^{2} t^{2}}{2 x}+o\left(\frac{1}{x}\right)\right),
$$

where

$$
m_{2}(r)=r^{2} \frac{\gamma^{\prime \prime}(r)}{\gamma(r)} .
$$

Finally, $F_{x}(t)$ converges to $\exp \left(\sigma^{2}(r) t^{2} / 2\right)$, with

$$
\sigma^{2}(r)=m(r)^{2}+m(r)-m_{2}(r) .
$$

Using the definitions of $m(r)$ and $m_{2}(r)$ as functions of $\gamma(r)$ and its derivatives, we obtain

$$
\sigma^{2}(r)=r\left(-r \frac{\gamma^{\prime}(r)}{\gamma(r)}\right)^{\prime}=r m^{\prime}(r) .
$$

Using the formula for $m(r)$ at the beginning of this section, we finally obtain

$$
\sigma^{2}(r)=\frac{1}{4} \sqrt{\frac{r}{(1+r)^{3}}}=\frac{u(1-u)}{(1+u)^{3}}=\sigma_{u}^{2} .
$$

This completes the proof.

\section{Conditional hitting times}

\subsection{Proof of Theorem 3}

We introduce the renewal process $\left(\zeta_{x}\right)_{x \geq 1}$ with increments $\left(\varepsilon_{x}\right)_{x \geq 1}$, that is,

$$
\zeta_{x}=\inf \left\{y \geq 1 ; \sigma_{y} \geq x\right\} .
$$

The usual central limit theorem for renewal processes states that $\left(\zeta_{x}-m x\right) / \sqrt{x}$ converges in distribution to a centered Gaussian variable whose variance is the variance $u(1-u)$ of every $\varepsilon_{x}$ divided by the cube of the mean $1+u$ of every $\varepsilon_{x}$, that is, $u(1-u) /(1+u)^{3}=\sigma_{u}^{2}$.

Our next lemma expresses the distribution of $\eta_{x}$ for every positive $x$ in terms of the distributions of the random variables $\left(\zeta_{z}\right)_{1 \leq z \leq x+1}$.

Lemma 7. For every positive $x$ and $y$,

$$
\mathrm{P}\left(\eta_{x}=y\right)=\frac{1+u}{1-(-u)^{x+1}} \sum_{z=0}^{x-1}(-u)^{z} \mathrm{P}\left(\zeta_{x+1-z}=y+1\right) .
$$

Proof. Let $x$ and $y$ denote positive integers. We begin with the fact that

$$
\left\{\zeta_{x+1}=y+1\right\}=\left\{\sigma_{y}=x\right\} \cup\left\{\sigma_{y}=x-1, \varepsilon_{y+1}=2\right\} ;
$$

hence,

$$
\mathrm{P}\left(\sigma_{y}=x\right)=\mathrm{P}\left(\zeta_{x+1}=y+1\right)-u \mathrm{P}\left(\sigma_{y}=x-1\right)
$$


Iterating this recursion, we obtain

$$
\mathrm{P}\left(H_{x}\right) \mathrm{P}\left(\eta_{x}=y\right)=\mathrm{P}\left(\sigma_{y}=x\right)=\sum_{z=0}^{x-1}(-u)^{z} \mathrm{P}\left(\zeta_{x+1-z}=y+1\right) .
$$

Summing over every positive value of $y$ and using the facts that $\mathrm{P}\left(\zeta_{z}=1\right)=0$ if $z \geq 3$ and $\mathrm{P}\left(\zeta_{2}=1\right)=u$, we obtain

$$
\mathrm{P}\left(H_{x}\right)=(-u)^{x-1}(1-u)+\sum_{z=0}^{x-2}(-u)^{z}=\frac{1-(-u)^{x+1}}{1+u} .
$$

This completes the proof.

Lemma 7, the fact that $|u|<1$, and the convergence of the distribution of $\left(\zeta_{x}-m x\right) / \sqrt{x}$ imply the same convergence for the distribution of $\left(\eta_{x}-m x\right) / \sqrt{x}$.

Finally, $\left(\xi_{x}-m x\right) / \sqrt{x},\left(\zeta_{x}-m x\right) / \sqrt{x}$, and $\left(\eta_{x}-m x\right) / \sqrt{x}$ all converge in distribution to the same limit, which is the centered Gaussian distribution with variance $\sigma_{u}^{2}$.

\subsection{Sharp bounds}

Lemma 8. For every positive $x$,

$$
\mathrm{E}\left(\eta_{x}\right)=\frac{x+1}{1+u} \frac{1+(-u)^{x+2}}{1-(-u)^{x+1}}-\frac{1+u^{2}}{(1+u)^{2}} .
$$

For instance,

$$
\mathrm{E}\left(\eta_{1}\right)=1, \quad \mathrm{E}\left(\eta_{2}\right)=2-\frac{u}{1-u(1-u)} .
$$

For every positive integer $x$, we can deduce from the exact formula above that

$$
\frac{x}{1+u}-\frac{2 u^{2}}{(1+u)^{2}} \leq \mathrm{E}\left(\eta_{x}\right) \leq \frac{x}{1+u}+\frac{2 u}{(1+u)^{2}} .
$$

The width of the interval delimited by the upper and the lower bounds of $\mathrm{E}\left(\eta_{x}\right)$ above is $2 u /(1+u) \leq 1$.

Bounds on $\mathrm{E}\left(\eta_{x}\right)$, depending on the parity of $x$, are as follows. For every odd $x$,

$$
\mathrm{E}\left(\eta_{x}\right) \geq \frac{x}{1+u}
$$

and, for every even $x$,

$$
\mathrm{E}\left(\eta_{x}\right) \leq \frac{x}{1+u}+\frac{u(1-u)}{1+u} \leq \frac{x+1 / 4}{1+u} .
$$

These refined bounds yield intervals around $\mathrm{E}\left(\eta_{x}\right)$, which depend on the parity of $x$, and whose width is always at most $2 u /(1+u)^{2} \leq \frac{1}{2}$.

Proof of Lemma 8. Fix a positive integer $x$, a real number $u$ in $(0,1)$, and let $r=\varrho(u)$. Let $p_{x}^{y}=\mathrm{P}\left(\sigma_{y}=x\right)$. Then $\eta_{x}(\mathrm{P})$ is proportional to the measure $\sum_{y} p_{x}^{y} \delta_{y}$ and, for every positive $y$,

$$
\sum_{x=y}^{2 y} p_{x}^{y} t^{x}=\left((1-u) t+u t^{2}\right)^{y}
$$


Hence, the distribution of $\eta_{x}$ is $\mu_{\eta}(r, x)$, where $\mu_{\eta}(r, x)=v_{\eta}(r, x) /\left|v_{\eta}(r, x)\right|$ and

$$
v_{\eta}(r, x)=\sum_{y=h(x)}^{x}\left(\begin{array}{c}
y \\
x-y
\end{array}\right) 4^{y} r^{y} \delta_{y} .
$$

When $u=0, r=\infty$ and $\mu_{\eta}(\infty, x)$ is the Dirac distribution at $x$. When $u=1, r=0$ and $\mu_{\eta}(0, x)$ is the Dirac distribution at $h(x)$. For the first values of $x$, the distributions $\mu_{\eta}(r, x)$ are as follows:

$$
\begin{gathered}
\mu_{\eta}(r, 1)=\delta_{1}, \quad \mu_{\eta}(r, 2)=\frac{\delta_{1}+4 r \delta_{2}}{1+4 r}, \quad \mu_{\eta}(r, 3)=\frac{2 \delta_{2}+4 r \delta_{3}}{2+4 r}, \\
\text { and } \quad \mu_{\eta}(r, 4)=\frac{\delta_{2}+12 r \delta_{3}+16 r^{2} \delta_{4}}{1+12 r+16 r^{2}} .
\end{gathered}
$$

This implies that, for every positive $x$,

$$
\mathrm{E}\left(\eta_{x}\right)=\frac{r g_{x}^{\prime}(r)}{g_{x}(r)}, \quad g_{x}(r)=\sum_{y=h(x)}^{x}\left(\begin{array}{c}
y \\
x-y
\end{array}\right) 4^{y} r^{y} .
$$

To study the generating functions $g_{x}$, we introduce $g_{0}(r)=1$ and

$$
G(r, z)=\sum_{x \geq 0} g_{x}(r) z^{x}
$$

Summing first over $y \leq x \leq 2 y$, then over $y \geq 0$, we obtain

$$
G(r, z)=\sum_{y \geq 0}(4 r z)^{y}(1+z)^{y}=\frac{1}{1-4 r z(1+z)}=C_{1}(r, z) .
$$

From the proof of Lemma 4 we know that the poles of $C_{1}(r, z)$ are $z=\gamma(r)$ and $z=-\gamma_{2}(r)$, with $\gamma_{2}(r)=\gamma(r)+1$; hence,

$$
G(r, z)=\frac{1}{\gamma(r)+\gamma_{2}(r)}\left(\frac{\gamma_{2}(r)}{1-z / \gamma(r)}+\frac{\gamma(r)}{1+z / \gamma_{2}(r)}\right) .
$$

This shows that, for every nonnegative $x$,

$$
g_{x}(r)=\frac{\gamma(r) \gamma_{2}(r)}{\gamma(r)+\gamma_{2}(r)}\left(\gamma(r)^{-(x+1)}-\left(-\gamma_{2}(r)\right)^{-(x+1)}\right) .
$$

From here, the expression of $\gamma(r)$ as a function of $r$ and tedious computations of derivatives yield the result.

\subsection{Comparison with a naïve estimator}

For a given value $u$ in $(0,1)$ and for a branching process $X_{0: \infty}$ with offspring distribution $(1-u) \delta_{1}+u \delta_{2}$, when $n$ converges to $\infty$,

$$
S_{n} \sim X_{n}\left(1+\frac{1}{1+u}+\frac{1}{(1+u)^{2}}+\cdots\right)=X_{n}\left(1+\frac{1}{u}\right) \quad \text { almost surely; }
$$


hence, $B\left(X_{0: \infty}\right)=u$ almost surely. The naïve pointwise prediction of the mean initial population conditional on $X_{1}=x$, namely $N_{u}(x)=x /(1+u)$, should be compared to the Bayesian prediction $\mathrm{E}_{u}\left(\xi_{x}\right)$ for $r=\varrho(u)$. For $x=2$, we obtain

$$
\frac{\mathrm{E}_{u}\left(\xi_{2}\right)}{N_{u}(2)}=\frac{\left(4 u+6(1-u)^{2}\right)(1+u)}{2\left(4 u+3(1-u)^{2}\right)} .
$$

This ratio is 1 when $u=0$ or $u=1$, greater than 1 for every $u$ in $\left(0, \frac{1}{3}\right)$, and smaller than 1 for every $u$ in $\left(\frac{1}{3}, 1\right)$. Hence, the naïve and Bayesian predictions cannot be easily compared, at least on $X_{1}=x$ for a given finite $x$.

\section{References}

Alfers, D. AND Dinges, H. (1984). A normal approximation for beta and gamma tail probabilities. Z. Wahrscheinlichkeitsth. 65, 399-420.

Jagers, P. AND Klebaner, F. (2003). Random variation and concentration effects in PCR. J. Theoret. Biol. 224, 299-304.

Kass, R. E. and Wasserman, L. A. (1996). The selection of prior distributions by formal rules. J. Amer. Statist. Assoc. 91, 1343-1370.

Klebaner, F. C. And Sagitov, S. (2002). The age of a Galton-Watson population with a geometric offspring distribution. J. Appl. Prob. 39, 816-828.

LALAM, N. (2007). Statistical inference for quantitative polymerase chain reaction using a hidden Markov model: a Bayesian approach. Statist. Appl. Genetics Molec. Biol. 6, Article 10.

LALAM, N. AND JACOB, C. (2007). Bayesian estimation for quantification by real-time polymerase chain reaction under a branching process model of the DNA molecules amplification process. Math. Popul. Stud. 14, 111-129.

Mendoza, M. And Gutiérrez-Peña, E. (2000). Bayesian conjugate analysis of the Galton-Watson process. Test 9, 149-171.

Molina, M., González, M. And Mota, M. (1998). Bayesian inference for bisexual Galton-Watson processes. Commun. Statist. Theory Meth. 27, 1055-1070.

Peccoud, J. AND JACOB, C. (1996). Theoretical uncertainty of measurements using quantitative polymerase chain reaction. Biophys. J. 71, 101-108.

PIAU, D. (2002). Mutation-replication statistics of polymerase chain reactions. J. Computational Biol. 9, 831-847.

PIAU, D. (2004). Immortal branching Markov processes: averaging properties and PCR applications. Ann. Prob. 32, 337-364.

PIAU, D. (2005). Confidence intervals for non-homogeneous branching processes and PCR reactions. Ann. Prob. 33, 674-702.

Prakasa RaO, B. L. S. (1992). Nonparametric estimation for Galton-Watson type process. Statist. Prob. Lett. 13, 287-293.

ScOTT, D. (1987). On posterior asymptotic normality and asymptotic normality of estimators for the Galton-Watson process. J. R. Statist. Soc. Ser. B 49, 209-214.

Sun, F. (1995). The polymerase chain reaction and branching processes. J. Computational Biol. 23, 3034-3040.

Weiss, G. AND von Haeseler, A. (1995). Modeling the polymerase chain reaction. J. Computational Biol. 2, $49-61$.

Weiss, G. AND von HAESEler, A. (1997). A coalescent approach to the polymerase chain reaction. Nucleic Acids Res. 25, 3082-3087. 